\title{
Intermittent Low-Dose Thalidomide Plus Dexamethasone as Maintenance Therapy in Patients with Multiple Myeloma
}

\author{
Galieni P1*, Caraffa P', Bigazzi C', Falcioni $S^{1}$, \\ Mazzotta S', Pezzoni V', Dalsass A ${ }^{1}$, Mestichelli \\ $F^{1}$, Camaioni E', Travaglini $F^{1}$, Vagnoni $A^{2}$, \\ Napoletano $\mathbf{M}^{2}$, Angelini $\mathrm{S}^{1}$ and Ruggieri $\mathbf{M}^{1}$ \\ ${ }^{1}$ Department of Hematology and Stem Cell \\ Transplantation Unit, C. e G. Mazzoni Hospital, Italy \\ ${ }^{2}$ Department of Pharmacy Services, C. e G. Mazzoni \\ Hospital, Ascoli Piceno, Italy \\ *Corresponding author: Piero Galieni, Department of \\ Hematology and Stem Cell Transplantation Unit, C. e G. \\ Mazzoni Hospital, via degli Iris n. 1, 63100 Ascoli Piceno, \\ Italy
}

Received: December 18, 2018; Accepted: February 08, 2019; Published: February 15, 2019

\begin{abstract}
In patients with Multiple Myeloma (MM), maintenance therapy with thalidomide helps maintain the results achieved through first-line treatment, improving Progression-Free Survival (PFS) and even Overall Survival (OS). However, when given continuously thalidomide causes various adverse events and high toxicity, thus patients have an increased risk of developing neuropathies and thromboembolic complications. We evaluated the efficacy and safety of a treatment protocol combining intermittent low-dose thalidomide with a monthly fixed dose of dexamethasone, in order to reduce toxicity and adverse events without stopping the maintenance therapy. Between 2003 and 2014, at our centre 62 patients with MM received intermittent low-dose thalidomide $(50 \mathrm{mg} / \mathrm{day}$ for 2 weeks per month) in combination with a fixed dose of dexamethasone (20mg/day for 4 days per month) as maintenance treatment, plus low-dose salicylate $(100 \mathrm{mg} / \mathrm{day})$ as antithrombotic prophylaxis. We observed positive effects on both OS and PFS, with a statistically significant advantage in patients with standard-risk cytogenetics compared to patients classified as high-risk (median OS of 70 months vs 34; median PFS of 41 months vs 22). Furthermore, our treatment protocol resulted in a remarkable reduction in toxicity and adverse events: peripheral neuropathy rate was $1.6 \%$ and no thromboembolic complications were recorded. Patients mainly experienced moderate fatigue (12.9\%) and constipation (3.2\%), which did not require dose reductions or discontinuation of therapy. Based on these data, intermittent lowdose thalidomide plus dexamethasone should be taken into consideration as maintenance regimen for $\mathrm{MM}$, especially when treating elderly patients with favorable iFISH.
\end{abstract}

Keywords: Multiple myeloma; Intermittent low-dose thalidomide; Reduced incidence of adverse events; Low toxicity; Elderly patients; Standard-risk cytogenetics

\section{Introduction}

Although with the use of novel agents it is possible to achieve a good response to first-line treatment, Multiple Myeloma (MM) is still incurable in almost all cases. Recurring periods of remission and relapse constitute a distinctive trait of $\mathrm{MM}$, but remission times gradually become shorter while relapses progressively become less sensitive to therapies, and patients inevitably die because of diseaserelated and/or treatment-related complications [1]. Maintenance therapy in MM aims to maintain and, if possible, improve the results of an effective induction therapy, performed with or without autologous hematopoietic stem-cell transplantation [2]. Thalidomide was the first immunomodulatory drug to be used as maintenance therapy in MM: patients receiving maintenance therapy with thalidomide, administered at various doses as a single agent or in combination with steroids, showed significant improvements in Progression-Free Survival (PFS) and to a lesser extent in Overall Survival (OS) compared with both transplant-eligible and transplant-ineligible patients receiving no maintenance therapy [3]. However, the use and efficacy of this treatment are limited, because continuous administration of thalidomide, albeit at low doses, until disease progression is associated with neurological and thromboembolic toxicities. Furthermore, it was observed that thalidomide maintenance therapy should not be administered to patients with high-risk cytogenetics, because in such patients the positive results were inferior to those achieved by patients classified as standard-risk [4].

Taking into account the findings of the first studies on thalidomide as maintenance regimen in patients with advanced MM [5] as well as the current studies on thalidomide maintenance therapy, and considering the well known side effects of thalidomide along with the ongoing clinical trials involving the use of thalidomide in both first-line treatment and maintenance protocol, at our centre patients diagnosed with MM (not enrolled in any other clinical trials) received maintenance therapy with intermittent low-dose thalidomide in combination with a monthly fixed dose of dexamethasone. Our primary aim was to evaluate whether a discontinuous administration of low-dose thalidomide, compared to the same regimen given continuously, would be equally effective in preventing disease progression but with less toxicity, in order for patients with MM to
Ann Hematol Oncol - Volume 6 Issue 2 - 2019

ISSN : 2375-7965 | www.austinpublishing group.com

Galieni et al. (C) All rights are reserved
Citation: Galieni P, Caraffa P, Bigazzi C, Falcioni S, Mazzotta S, Pezzoni V, et al. Intermittent Low-Dose Thalidomide Plus Dexamethasone as Maintenance Therapy in Patients with Multiple Myeloma. Ann Hematol Oncol. 2019; 6(2): 1235. 


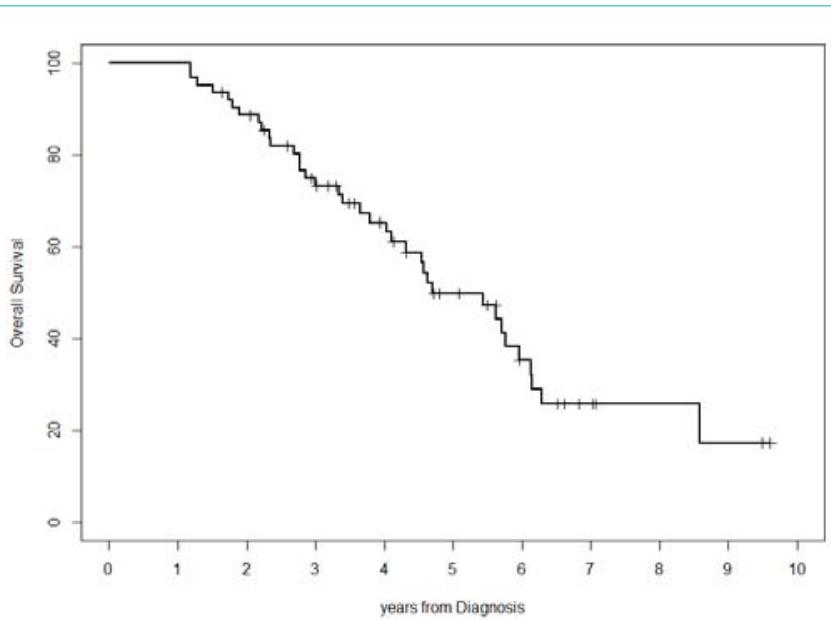

Figure 1: Overall Survival.

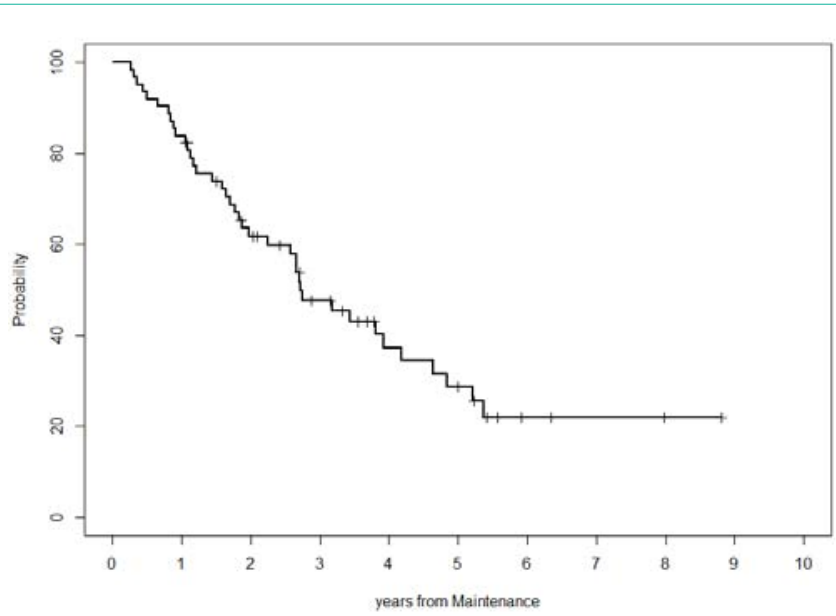

Figure 2: Progression-Free Survival.

Progression-free survival: $62 \%$ (std. error $6 \%$ ) at 2 years from the start of maintenance therapy, $29 \%$ (std. error $7 \%$ ) at 5 years from the start of maintenance therapy, and $22 \%$ (std. error: $7 \%$ ) at approximately 9 years from the start of maintenance therapy (8 years and 10 months).

Median duration of survival: 2 years and 8 months.

receive maintenance therapy until relapse without any interruptions due to treatment-related complications.

\section{Patients and Methods}

\section{Study design and treatment protocol}

At our centre, between January 2003 and December 2014, 62 patients diagnosed with symptomatic MM, not enrolled in any other clinical trials and with at least a partial response achieved after induction therapy, received maintenance treatment with intermittent low-dose thalidomide in combination with a monthly fixed dose of dexamethasone.

Diagnosis of MM was based on the clinical diagnostic criteria established by the International Myeloma Working Group [6]. Patients' response to induction therapy and maintenance treatment was assessed according to the response criteria defined by the International Myeloma Working Group [7]. Toxicities were graded using the National Cancer Institute Common Toxicity Criteria.

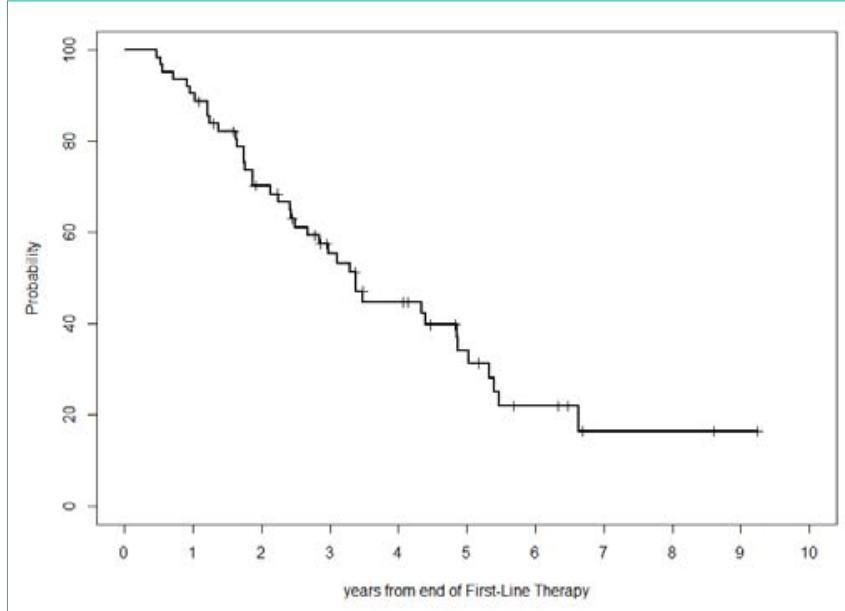

Figure 3: Duration of Response (Time to Next Treatment).

Time to Next Treatment: $70 \%$ (std. error $6 \%$ ) at 2 years from the end of firstline therapy, $34 \%$ (std. error $7 \%$ ) at 5 years from the end of first-line therapy, and $16 \%$ (std. error: $7 \%$ ) at approximately 9 years from the end of first-line therapy ( 9 years and 3 months). Median duration of response: 3 years and 5 months (at 3 years and 5 months from the end of first-line therapy, $50 \%$ of patients did not need retreatment).

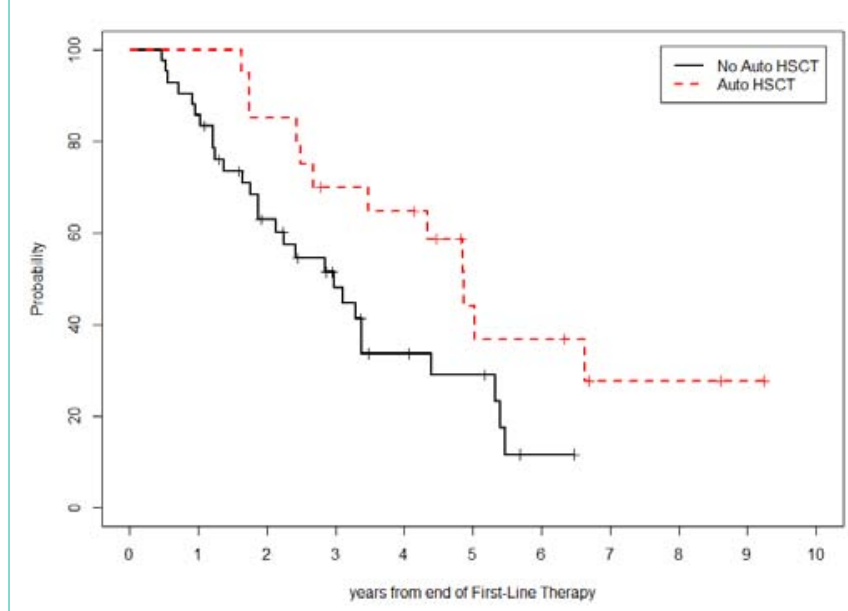

Figure 4: Time to Next Treatment Stratified By Asct (Yes Vs No).

NO ASCT: Time to Next Treatment: $63 \%$ (std. error $8 \%$ ) at 2 years from the end of first-line therapy, $29 \%$ (std. error $8 \%$ ) at 5 years from the end of firstline therapy, and $11 \%$ (std. error $7 \%$ ) at approximately 6 years from the end of first-line therapy (6 years and 4 months); Median duration of response: 3 years.

ASCT: Time to Next Treatment: $85 \%$ (std. error $8 \%$ ) at 2 years from the end of first-line therapy, $44 \%$ (std. error $12 \%$ ) at 5 years from the end of first-line therapy, and $27 \%$ (std. error: $12 \%$ ) at approximately 9 years from the end of first-line therapy (9 years and 3 months); Median duration of response: 5 years.

Log-Rank test is significant ( $p$-value=0.033).

This study was undertaken in conformity with the Declaration of Helsinki and written informed consent was provided by all patients before starting treatment.

Maintenance therapy consisted of thalidomide $50 \mathrm{mg} /$ day for 2 weeks per month (on days 1 through 14), and dexamethasone 20mg/ day for 4 days per month (on days 1 through 4 ).

Thalidomide was provided by the C. e G. Mazzoni Hospital 


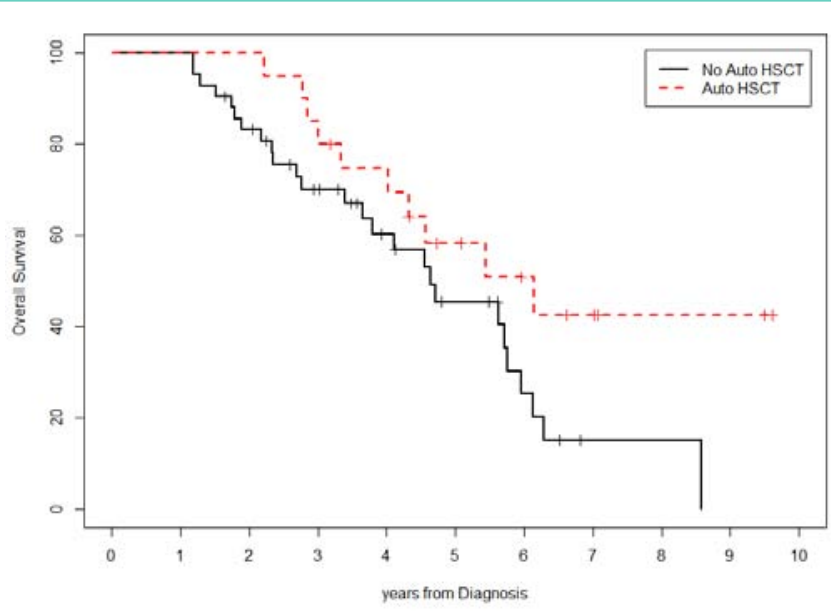

Figure 5: Os Stratified by Asct (Yes Vs No).

NO ASCT: Overall Survival: $83 \%$ (std. error $6 \%$ ) at 2 years from diagnosis, $45 \%$ (std. error $9 \%$ ) at 5 years from diagnosis, and $0 \%$ at approximately 9 years from diagnosis ( 8 years and 6 months); Median duration of survival: 4 years and 8 months.

ASCT: Overall Survival: $100 \%$ at 2 years from diagnosis, $58 \%$ (std. error $11 \%$ ) at 5 years from diagnosis, and $42 \%$ (std. error: 13\%) at approximately 10 years from diagnosis ( 9 years and 7 months); Median duration of survival: 6 years and 2 months.

Log-Rank test is not significant ( $p$-value $=0.0667$ ), there is a trend toward significance.

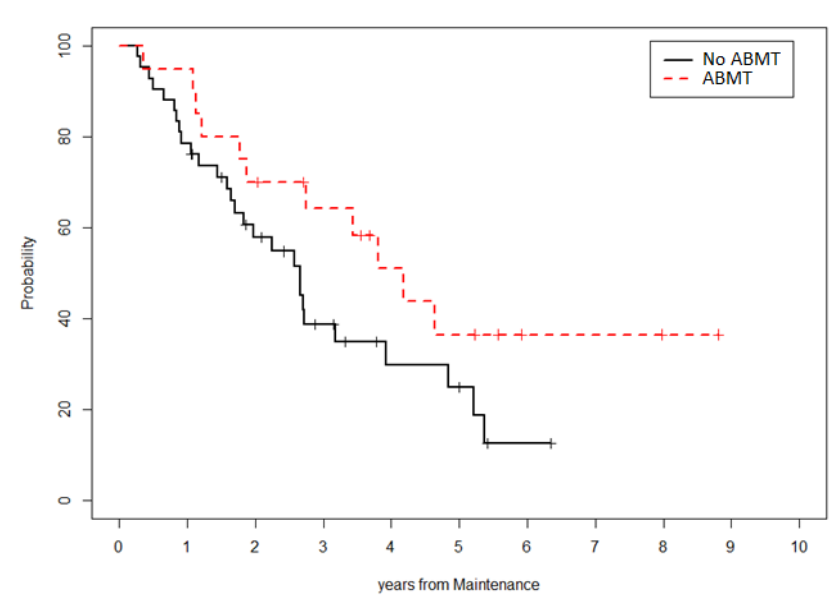

Figure 6: Pfs Stratified By Asct (Yes Vs No).

NO ASCT: Progression-Free Survival: $58 \%$ (std. error $9 \%$ ) at 2 years from the start of maintenance therapy, $25 \%$ (std. error $8 \%$ ) at 5 years from the start of maintenance therapy, and $12 \%$ (std. error $7 \%$ ) at approximately 6 years from the start of maintenance therapy (6 years and 4 months); Median duration of survival: 2 years and 8 months.

ASCT: Progression-Free Survival: 70\% (std. error 10\%) at 2 years from the start of maintenance therapy and $37 \%$ (std. error $12 \%$ ) at approximately 9 years from the start of maintenance therapy (8 years and 6 months); Median Duration of survival: 4 years and 2 months. Log-Rank test is not significant $(p$-value $=0.085)$

Pharmacy as a galenic compound, prepared as size 2 hard gelatin capsules containing thalidomide 50mg plus excipient type B (composition: pregelatinized maize starch $97,5 \%$ as diluentdisintegrant; magnesium stearate $1,5 \%$ as lubricant; micronized silica $0,5 \%$ as diluent; micronized talc $0,5 \%$ as diluent-lubricant), free of lactose and hence suitable for all patients.

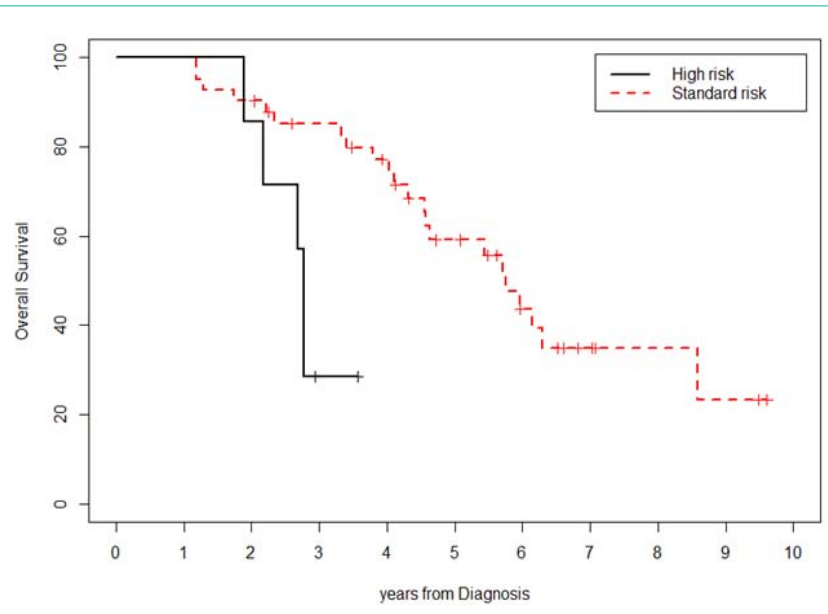

Figure 7: Os Stratified By Cytogenetics (Standard Risk Vs High Risk). Log-Rank Test is significant ( $p$-value $=0.0028$ ).

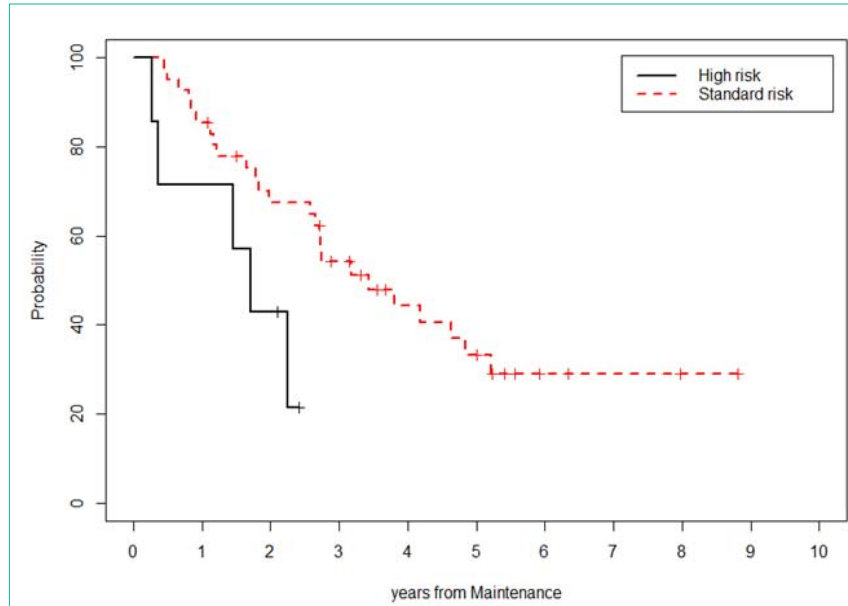

Figure 8: Pfs Stratified By Cytogenetics (Standard Risk Vs High Risk). Standard: Progression-Free Survival: 29\% (std. error 8\%) at 9 years from maintenance; Median Duration of Survival: 3 years and 5 months. High: Progression-Free Survival: 21\% (std. error 18\%) at 2 years and 9 months from maintenance; Median Duration of Survival: 1 year and 10 months.

Log-Rank Test is significant ( $p$-value=0.0329).

Antithrombotic prophylaxis with low-dose salicylate $(100 \mathrm{mg} /$ day) was given to all patients, and maintenance treatment was administered until disease progression and/or intolerance. Follow-up occurred every 3 months, with regular physical examinations preceded by laboratory tests (complete blood count, creatinine, calcium, total serum protein test and serum protein electrophoresis, serum free light chains assay, 24-hour urine total protein concentration plus Bence Jones protein test and, for patients in complete remission, serum/urine immunofixation). Bone marrow biopsy evaluation as well as X-ray, Computed Tomography (CT), Positron Emission Tomography (PET) and Magnetic Resonance Imaging (MRI) were performed in case of clinical or laboratory evidence of disease progression.

\section{Statistical analysis}

A descriptive analysis of all variables was performed including 


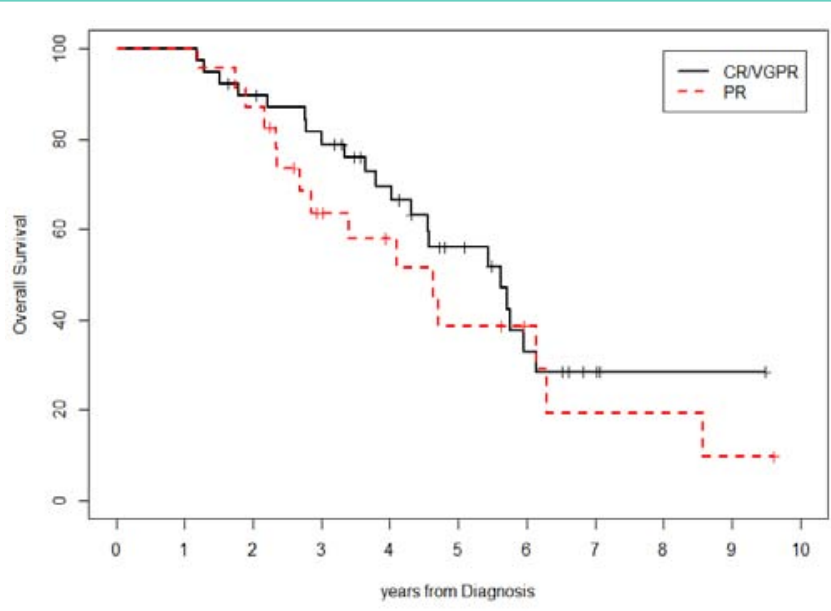

Figure 9: Os Stratified By Disease Status at Maintenance (Cr/Vgpr Vs Pr) CR/VGPR: Overall Survival: $28.2 \%$ (std. error $9 \%$ ) at 9 years and 6 months from diagnosis; Median Duration of Survival: 5 years and 7 months.

PR: Overall Survival: $9.6 \%$ (std. error $9 \%$ ) at 9 years and 7 months from diagnosis; Median Duration of Survival: 4 years and 6 months.

Log-Rank Test is not significant ( $p$-value $=0.067)$.

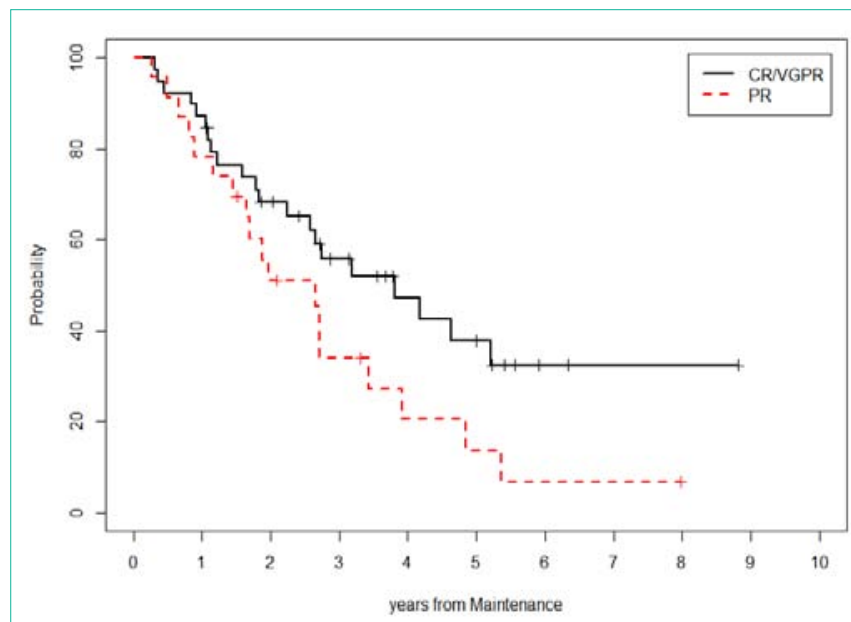

Figure 10: Pfs Stratified By Disease Status at Maintenance (Cr/Vgpr Vs Pr) CR/VGPR: Progression-Free Survival: $32.5 \%$ (std. error 9\%) at 8 years and 10 months from maintenance; Median Duration of Survival: 3 years and 10 months.

PR: Progression-Free Survival: $7 \%$ (std. error $6 \%$ ) at 8 years from maintenance; Median Duration of Survival: 2 years and 8 months.

Log-Rank Test is not significant ( $p$-value $=0.085$ )

mean, median, standard deviation, range, minimum and maximum value for continuous variables, absolute and relative frequencies for categorical variables. Using parametric and multiparametric statistical procedures (chi-square test, Fisher's exact test and Spearman's rank correlation coefficient), the possible interdependence between two or more variables was evaluated and a $p$ value of $\leq 0.05$ was considered significant. OS and PFS were estimated using the Kaplan-Meier product limit method [8] and the curves of various subgroups were compared using the log-rank test [9]. OS was measured from the date of diagnosis to death from any cause, with censoring performed at the date of last contact. PFS was measured from the start of maintenance therapy to relapse, progression, or death from any cause, with censoring performed at the date of last contact. The analysis was
Table 1: Baseline Characteristics of Patients enrolled in the Trial.

\begin{tabular}{|c|c|c|}
\hline \multicolumn{2}{|c|}{ Median age, y (range) } & \multirow[t]{2}{*}{$74(44-86)$} \\
\hline Age, $\mathrm{n}(\%)$ & & \\
\hline & $<65 y$ & $11(18)$ \\
\hline & $66-70 y$ & $7(11)$ \\
\hline & $>70 y$ & $44(71)$ \\
\hline \multicolumn{3}{|l|}{ Sex, n (\%) } \\
\hline & Male & $36(58)$ \\
\hline & Female & $26(42)$ \\
\hline \multicolumn{3}{|c|}{ M-Protein, n (\%) } \\
\hline & $\lg A$ & $13(21)$ \\
\hline & $\lg G$ & $30(48)$ \\
\hline & Micromolecular & $17(28)$ \\
\hline & Non-secretory & $2(3)$ \\
\hline \multicolumn{3}{|c|}{ ISS Stage, n (\%) } \\
\hline & I & $13(21)$ \\
\hline & II & $20(32)$ \\
\hline & III & $29(47)$ \\
\hline \multicolumn{3}{|c|}{ DS Stage, n (\%) } \\
\hline & I A & $4(7)$ \\
\hline & II A & $12(19)$ \\
\hline & III A & $34(55)$ \\
\hline & III B & $12(19)$ \\
\hline \multicolumn{3}{|c|}{ Cytogenetic Risk, n (\%) } \\
\hline & Standard & $41(66)$ \\
\hline & High & $7(11)$ \\
\hline & Unknown & $14(23)$ \\
\hline
\end{tabular}

conducted using two statistical software packages: SAS version 9.1.3 (http://www.sas.com/en_us/home.html) and R Version 2.15.3 (https://cran.r-project.org/).

\section{Results}

\section{Patients' characteristics}

Sixty-two patients ( 36 men and 26 women) with a median age at diagnosis of 74 years (range 44-86) were enrolled in this trial. Clinical features of patients are reported in Table 1. Twelve patients (19\%) had previously been diagnosed with Monoclonal Gammopathy of Undetermined Significance (MGUS) and 5 patients (8\%) with Smoldering Multiple Myeloma (SMM).

Patients were stratified according to Durie-Salmon (DS) staging system and International Staging System (ISS) criteria, and also on the basis of MM isotype and cytogenetic risk profile, induction therapy and response to treatment, as shown in Table 1,2.

Using the DS staging system, 46 patients (75\%) were stage III, 12 patients (19\%) were stage II and 4 patients (6\%) were stage I; renal insufficiency was present in only 12 patients (19\%).

Following the ISS criteria, 29 patients (47\%) were stage III, 20 patients (32\%) were stage II and 13 patients (21\%) were stage I; with 
Table 2: First-Line Treatments and Response.

\begin{tabular}{|l|l|c|}
\hline First-Line Therapy, $n$ (\%) & & \\
\hline & VTD + ASCT & $10(16)$ \\
\hline & ThaDD + ASCT & $7(11)$ \\
\hline & Other + ASCT & $3(5)$ \\
\hline & MPT & $18(29)$ \\
\hline ASCT, n (\%) & ThaDD & $17(28)$ \\
\hline & Other & $7(11)$ \\
\hline & & $20(32)$ \\
\hline Melphalan dose prior to ASCT, $n(\%)$ & Yes & $42(68)$ \\
\hline & No & \\
\hline & $200 \mathrm{mg} / \mathrm{mq}$ & $11(61)$ \\
\hline Disease Status at Maintenance, $\mathrm{n}(\%)$ & $140 \mathrm{mg} / \mathrm{mq}$ & $7(39)$ \\
\hline & & $21(34)$ \\
\hline & CR & 237 (29) \\
\hline & VGPR & \\
\hline & PR & \\
\hline
\end{tabular}

Table 3: Clinical Course of Patients.

\begin{tabular}{|l|c|c|c|c|}
\hline & $\begin{array}{c}\text { Continuous } \\
\text { Treatment }\end{array}$ & Relapsed & Alive & Dead \\
\hline $\begin{array}{l}\text { Conventional Treatment, } \\
\mathrm{n}(\%)\end{array}$ & $13(31)$ & $21(50)$ & $16(38)$ & $26(62)$ \\
\hline ASCT, n (\%) & $8(40)$ & $6(30)$ & $10(50)$ & $10(50)$ \\
\hline Total, n (\%) & $21(34)$ & $27(43)$ & $26(42)$ & $36(58)$ \\
\hline
\end{tabular}

Table 4: Incidence and Grade of Adverse Events.

\begin{tabular}{|l|c|c|c|}
\hline & Grade 1 & Grade 2 & Grade 3-4 \\
\hline Fatigue, n (\%) & $0(0)$ & $8(12.9)$ & $0(0)$ \\
\hline Constipation, n (\%) & $0(0)$ & $2(3.2)$ & $0(0)$ \\
\hline Atrial Fibrillation, n (\%) & $1(1.6)$ & $0(0)$ & $0(0)$ \\
\hline Paresthesia, n (\%) & $1(1.6)$ & $0(0)$ & $0(0)$ \\
\hline Psychomotor Agitation, n (\%) & $1(1.6)$ & $0(0)$ & $0(0)$ \\
\hline
\end{tabular}

regard to the cytogenetic analysis, 41 patients $(66.1 \%)$ were classified as standard-risk and 7 patients (11.3\%) were classified as high-risk, while in 14 patients (22.6\%) cytogenetics were not evaluable.

Treatment regimens given to patients prior to maintenance therapy are listed in Table 2 . Of the 62 patients, $42(68 \%)$ had received chemotherapy alone; the remaining 20 patients (32\%), 11 (18\%) aged $<65$ years and $9(14 \%)$ aged 66-70 years, had undergone cytoreductive therapy followed by Autologous Stem-Cell Transplantation (ASCT): of these, 50\% had received induction therapy with BortezomibThalidomide-Dexamethasone (VTD), 35\% had been administered Thalidomide-Dexamethasone-Pegylated Liposomal Doxorubicin (ThaDD) and 15\% had been treated with other drug combinations such as Bortezomib-Dexamethasone (VD), Thalidomide-Dexamethasone (TD) or Vincristine-Doxorubicin-Dexamethasone (VAD). Younger patients had undergone single or double ASCT conditioned with melphalan $200 \mathrm{mg} / \mathrm{m}^{2}$; patients aged $>65$ years had received a single ASCT conditioned with melphalan $140 \mathrm{mg} / \mathrm{m}^{2}$.
Of 42 patients $(71 \%)$ aged $>70$ years, $43 \%$ had received $6-8$ cycles of Melphalan-Prednisone-Thalidomide (MPT), 40\% had been given ThaDD and 7\% had been treated with other chemotherapy regimens like Bortezomib-Melphalan-Prednisone (VMP), VTD or VD.

According to the criteria established by the International Myeloma Working Group, before starting maintenance therapy 7 patients (11\%) had achieved a stringent Complete Response (sCR) to treatment, 11 patients (18\%) a Complete Response (CR), 21 patients (34\%) a Very Good Partial Response (VGPR) and 23 patients (37\%) a Partial Response (PR). Patients' response status after first-line treatment is shown in Table 2.

\section{Clinical course}

Twenty-seven patients out of 62 (43\%) relapsed during maintenance therapy, but only 2 of them did not receive a second-line of treatment because of death. The clinical course of patients, from the start of maintenance therapy, is reported in Table 3.

At last follow-up (31/03/2017), 26 patients (42\%) were alive: of these, $21(80 \%)$ continue to receive maintenance therapy. Of the 62 patients, 36 (58\%) died; the most common causes of death were relapse/disease progression in $61 \%$ of cases, cardiovascular events (22\%) and infections (11\%), while 6\% of patients died from unknown causes. The median duration of maintenance therapy was 30 months (range 4-99 months).

The median follow-up time was 5 years from diagnosis and 4 years from the start of maintenance therapy.

\section{Survival and Duration of Response}

At 10 years from diagnosis, OS was $17 \%$ with a median duration of 56 months. At 9 years from the start of maintenance therapy, PFS was $22 \%$ with a median duration of 32 months. OS and PFS are shown in Figure 1,2 respectively.

The median duration of response was 41 months and at 9 years from the end of first-line therapy retreatment was not needed in $16 \%$ of patients (Figure 3). A statistically significant advantage in Time To Next Treatment (TTNT) was observed in patients who had undergone ASCT compared with patients that were not eligible for transplant (Figure 4), while there were no significant differences in OS and PFS between the two groups (Figure 5\&6).

A statistically significant advantage in OS and PFS was also observed in patients with standard-risk cytogenetics compared to patients classified as high-risk (median OS of 70 months $v s 34$; median PFS of 41 months $v s 22, \mathrm{p}=0.03$ ), as shown in Figure $7 \& 8$ respectively.

There were no significant differences in OS and PFS based on patients' response to first line therapy (Figure 9\&10).

\section{Safety}

Incidence and grade of adverse events, reported by patients, are listed in Table 4: they were mild to moderate, mainly grade II fatigue (12.9\%) and constipation (3.2\%), and in no case it was necessary to stop the therapy. The results also indicate a very low rate of peripheral neuropathy (1.6\%), which occurred as grade I paresthesia. No thromboembolic events and no grade III-IV toxicities were recorded. No patient has developed a second neoplasm. 
Table 5: Thalidomide Maintenance-After ASCT.

\begin{tabular}{|c|c|c|c|c|c|}
\hline Study & $\begin{array}{l}\text { Median age, y } \\
\text { (no. of patients) }\end{array}$ & Maintenance and Duration of Treatment & EFS or PFS & OS & Thalidomide Tolerance \\
\hline $\begin{array}{l}\text { IFM 99-02: } \\
\text { Attal et al [15] }\end{array}$ & $\begin{array}{l}\text { Mean } 59 \pm 8 \\
(\mathrm{~N}=597)\end{array}$ & $\begin{array}{l}\text { (A) Thalidomide } 400 \text { mg/day until PD } \\
\text { Pamidronate } 90 \text { mg every } 4 \text { wks until PD B) } \\
\text { Pamidronate } 90 \text { mg every } 4 \text { wks until PD }\end{array}$ & $\begin{array}{l}3-y \text { EFS: } \\
\text { (A) } 52 \% \\
\text { (B) } 37 \% \\
\text { (C) } 36 \% \\
P<.009\end{array}$ & $\begin{array}{l}4-y \text { OS: (A) } 87 \% \\
\text { (B) } 74 \% \text { (C) } 77 \% \\
P<.04\end{array}$ & $\begin{array}{l}39 \% \text { stopped thalidomide because of side } \\
\text { effects, mostly PNP; all grades of PNP } \\
68 \% \text {, grades } 3 \text { or } 4: 7 \%\end{array}$ \\
\hline $\begin{array}{l}\text { ALLG MM6: } \\
\text { Spencer et al [18] }\end{array}$ & $\begin{array}{l}\leq 70 \\
(\mathrm{~N}=243)\end{array}$ & $\begin{array}{l}\text { (A) Thalidomide } 100-200 \mathrm{mg} / \text { day for } 12 \\
\text { mo Prednisolone } 50 \mathrm{mg} \text { on alternate days } \\
\text { until PD } \\
\text { (B) Prednisolone } 50 \mathrm{mg} \text { on alternate days } \\
\text { until PD }\end{array}$ & $\begin{array}{l}3-y \text { PFS: } \\
\text { (A) } 42 \% \\
\text { (B) } 23 \% \\
P<.001\end{array}$ & $\begin{array}{l}\text { 3-y OS: (A) } 86 \% \\
\text { (B) } 75 \% P=.004\end{array}$ & $\begin{array}{l}30 \% \text { stopped therapy because of } \\
\text { intolerance (mostly PNP), } 10 \% \text { grades } 3 \\
\text { or } 4 \text { PNP, and } 9 \% \text { stopped because of PD }\end{array}$ \\
\hline $\begin{array}{l}\text { MRC Myeloma IX: } \\
\text { Morgan et al [4] }\end{array}$ & $\begin{array}{l}\text { Intensive, } 59 \\
(\mathrm{~N}=493)\end{array}$ & (A) Thalidomide 50-100 mg/day until PD & $\begin{array}{l}\text { PFS: } \\
\text { (A) } 30 \text { mo }\end{array}$ & 3-year OS: (A) $75 \%$ & $\begin{array}{l}52.2 \% \text { discontinued maintenance before } \\
\text { PD because of adverse events; median } \\
\text { duration of treatment: } 7 \text { mo }\end{array}$ \\
\hline $\begin{array}{l}\text { TT2: } \\
\text { Barlogie et al [14] }\end{array}$ & $\begin{array}{l}\leq 75, \text { median } \mathrm{NA} \\
(\mathrm{N}=668) \\
\text { Median follow- } \\
\text { up: } 42 \text { mo }\end{array}$ & $\begin{array}{l}\text { (A) Thalidomide } 100 \mathrm{mg} / \text { day during the first } \\
\text { year, thereafter } 50 \mathrm{mg} \text { on alternate days, } \\
\text { until PD } \\
\text { (B) None }\end{array}$ & $\begin{array}{l}\text { 4-y EFS: } \\
\text { (A) } 65 \% \\
\text { (B) } 44 \% \\
P=.01\end{array}$ & $\begin{array}{l}\text { OS: } \\
\text { (A) Not stated } \\
\text { (B) Not stated } \\
P=.9\end{array}$ & $30 \%$ stopped thalidomide within 2 y \\
\hline $\begin{array}{l}\text { HOVON-50: } \\
\text { Lokhorst et al [17] }\end{array}$ & $56(N=556)$ & (A) Thalidomide $50 \mathrm{mg} /$ day until & EFS: & Median: & PNP grades 2-4: 50\% \\
\hline Lokhorst et al [17] & & PD (B) IFN- $\alpha 3$ MU 3 times weekly until PD & $\begin{array}{l}\text { (A) } 34 \mathrm{mo} \\
\text { (B) } 22 \mathrm{mo} \\
P<.001 \mathrm{PFS}: \\
\text { (A) } 34 \mathrm{mo} \\
\text { (B) } 25 \mathrm{mo} \\
P<.001\end{array}$ & $\begin{array}{l}\text { (A) } 73 \mathrm{mo}(\mathrm{B}) 60 \\
\mathrm{mo} \\
\mathrm{P}=.77\end{array}$ & $\begin{array}{l}\text { Treatment discontinued or dose reduced: } \\
58 \%\end{array}$ \\
\hline $\begin{array}{l}\text { NCIC-CTG MY.10: } \\
\text { Stewart et al [16] }\end{array}$ & $58(N=332)$ & $\begin{array}{l}\text { (A) Thalidomide } 200 \mathrm{mg} / \text { day and } \\
\text { prednisone } 50 \mathrm{mg} \text { on alternate-day until PD } \\
\text { (B) None }\end{array}$ & $\begin{array}{l}\text { 4-y PFS: } \\
\text { (A) } 32 \% \text { (B) } 14 \% \\
P<.0001\end{array}$ & $\begin{array}{l}\text { 4-y OS: (A) } 68 \% \\
\text { (B) } 60 \% P=.18\end{array}$ & $\begin{array}{l}\text { PNP grades } 3-4: 9.6 \% \\
\text { VTE: } 7.3 \% \\
\text { Infection: } 23.6 \%\end{array}$ \\
\hline
\end{tabular}

Table 6: Thalidomide Maintenance-No ASCT.

\begin{tabular}{|c|c|c|c|c|c|}
\hline Study & $\begin{array}{l}\text { Induction } \\
\text { Therapy }\end{array}$ & Maintenance & PFS/EFS & OS & Thalidomide Tolerance \\
\hline $\begin{array}{l}\text { Palumbo et al } \\
\text { [19] }\end{array}$ & MP vs MPT & $\begin{array}{l}\text { No Maintenance vs Thalidomide } \\
(100 \mathrm{mg} / \mathrm{d})\end{array}$ & $\begin{array}{l}\text { PFS median: } 14,5 \text { vs } \\
21,8 \text { mo } P=.0004\end{array}$ & $\begin{array}{l}\text { OS median: } 47,6 \text { vS } \\
45 \text { mo } P=.79\end{array}$ & PNP Grade 3-4: 14\%. Thromboembolism 11\% \\
\hline $\begin{array}{l}\text { Wijermans et } \\
\text { al [20] }\end{array}$ & MP vs MPT & $\begin{array}{l}\text { No Maintenance vs Thalidomide } \\
(50 \mathrm{mg} / \mathrm{d}) \mathrm{P}<.001\end{array}$ & $\begin{array}{l}\text { EFS median: } 9 \text { vs } \\
13 \text { mo }\end{array}$ & $\begin{array}{l}\text { OS median: } 31 \text { vs } 40 \\
\text { mo } P=.05\end{array}$ & PNP Grade $\geq 2: 54 \%$. Thromboembolism 10\% \\
\hline Ludwig et al [21] & MP vs TD & IFN vs IFN-Thalidomide $(100 \mathrm{mg} / \mathrm{d})$ & $\begin{array}{l}\text { PFS median: } 16,7 \text { vs } \\
20,7 \text { mo } P=.0068\end{array}$ & $\begin{array}{l}\text { OS median: } 51,4 \text { vs } \\
52,6 \text { mo } P=.81\end{array}$ & $\begin{array}{l}\text { PNP Grade 1-2: } 62 \% \text { PNP Grade 3-4: } 7 \% \\
\text { Constipation Grade 1-2: } 28 \% \text { Constipation } \\
\text { Grade 3-4: } 5 \%\end{array}$ \\
\hline Offidani et al [22] & ThaDD & $\begin{array}{l}\text { IFN-Dexamethasone } v s \\
\text { Dexamethasone-Thalidomide }(100 \\
\mathrm{mg} / \mathrm{d})\end{array}$ & $\begin{array}{l}2-y \text { PFS: } 32 \% \text { vs } \\
63 \% P=0.024\end{array}$ & $\begin{array}{l}\text { 2-y OS: } 68 \% \text { vs } 84 \% \\
P=.03\end{array}$ & $\begin{array}{l}\text { PNP Grade 3-4: 6\%. Constipation Grade 3-4: } \\
4 \%\end{array}$ \\
\hline
\end{tabular}

\section{Discussion}

Over the last 20 years, considerable improvements have been made in the treatment of patients with MM; in particular, the use of ASCT and novel agents have determined a significant increase in OS and PFS.

However, despite that, MM remains incurable. Furthermore, patients have a high probability of relapsing due to clonal heterogeneity and genomic instability, which are characteristic features of the disease.

The primary objective of maintenance therapy is to increase both the duration of response to previous treatments and patients' survival.

Among the first therapeutic options in maintenance regimen, steroids have been proven to successfully extend the duration of response to previous therapies, but with limited effects on survival, as reported in some studies $[10,11]$.

Interferon (IFN) also played an important role, since initial trials produced encouraging results in terms of prolonged survival, especially in patients responding to induction therapy $(\mathrm{P}=0.0352)$
[12]. However, the next studies with a longer follow-up did not confirm such data, showing only a marginal benefit in terms of PFS and OS at the cost of numerous toxicities and adverse events [13]. The final results of a phase III trial eventually proved that IFN maintenance therapy was indeed ineffective regardless of the treatments used, namely ASCT or standard-dose chemotherapy [14].

The introduction of thalidomide, a non-myelotoxic agent with the advantage of oral administration, into the treatment of MM prompted numerous trials to evaluate its efficacy as maintenance therapy in both younger patients who had undergone ASCT and older/transplant-ineligible patients.

There are many trials assessing thalidomide maintenance therapy after ASCT (Table 5)

Specifically, the 9902 study by the Inter-Groupe Francophone du Myélome (IFM) showed that a maintenance therapy including thalidomide at $400 \mathrm{mg} / \mathrm{day}$, compared to both pamidronate maintenance and no maintenance, produced better results in terms of Event-Free Survival (EFS), but only in those patients who had achieved less than a VGPR after ASCT. The median duration of 
treatment was just 15 months and neuropathy reached 68\% [15].

The National Cancer Institute of Canada CTG (NCIC-CTG) MY.10 trial reported that, in comparison with the observation group, a significant improvement in PFS was seen in patients receiving maintenance therapy with thalidomide $(200 \mathrm{mg} /$ day), but it was associated with a worse health-related quality of life [16].

In the HOVON-50 study, a maintenance treatment with a remarkably lower dosage of thalidomide $(50 \mathrm{mg} /$ day) significantly improved both EFS and PFS compared to interferon maintenance; however, polyneuropathy occurred in almost $50 \%$ of patients, and in $58 \%$ of patients thalidomide had to be stopped or reduced due to toxicity [17].

In contrast to the others, the ALLG MM6 study showed an improved OS in patients receiving maintenance therapy with the addition of thalidomide (100-200mg/day) for 12 months; however, despite its limited duration, $30 \%$ of patients had to discontinue treatment due to adverse events, mainly neuropathy [18].

The MRC Myeloma IX study compared thalidomide maintenance with no maintenance. An updated analysis reported a similar result in terms of OS in both groups $(p=0.4)$ and a significant advantage in terms of PFS in the group receiving thalidomide maintenance $(\mathrm{p}<0.001)$. However, a subgroup analysis showed that this advantage was observed only in patients with favorable iFISH, while in patients with adverse iFISH thalidomide maintenance therapy had no beneficial effect on PFS and resulted in even worse OS ( $p=0.009)$ [4].

There are also several studies on thalidomide as maintenance treatment in elderly and transplant-ineligible patients (Table 6). In particular, seven trials compared Melphalan-Prednisone (MP) regimen with MPT regimen, and in 4 of them thalidomide was given as maintenance therapy after MPT induction treatment.

Both GIMEMA (thalidomide at $100 \mathrm{mg} /$ day) and HOVON 49 (thalidomide at $50 \mathrm{mg} /$ day) trials showed an improvement in terms of PFS in patients receiving thalidomide maintenance after induction therapy with MPT, while an advantage in terms of OS was observed only in the latter study $[19,20]$.

In another trial, after completing induction therapy with either MP or TD, elderly patients received maintenance therapy with either thalidomide-interferon (Thal-IFN) or IFN alone: the study observed that Thal-IFN maintenance produced a significantly longer PFS compared to IFN maintenance $(\mathrm{P}=0.0068)$, while there was no benefit in $\mathrm{OS}(\mathrm{P}=0.81)$ [21].

The combination of thalidomide and dexamethasone as maintenance therapy was compared to Interferon and Dexamethasone (ID) after induction therapy with ThaDD in newly diagnosed transplant-ineligible patients and relapsed patients: a significantly better result in terms of PFS and OS was seen in both categories of patients assigned to the TD maintenance group but, in this trial as well, only patients who had achieved less than a VGPR after induction therapy benefited from thalidomide maintenance [22].

In our patients, treated with intermittent low-dose thalidomide, PFS and OS are comparable with those reported in the abovementioned studies in addition to a better toxicity profile.
If PFS and OS are stratified on the basis of high-dose therapy plus ASCT vs standard-dose chemotherapy, there are no statistically significant differences between the two arms, while median PFS observed in elderly patients is superior to that reported in previously published data [19-21], and PFS at 2 years is comparable with that seen in the Italian study [22]. We also observed that median OS is superior to that documented in the above-mentioned reports.

In younger patients, the longest median PFS (34 months) registered in the HOVON-50 study [17] is inferior to median PFS observed in our trial (50 months). Furthermore, in our patients PFS at 6 years from ASCT is 37\%, slightly inferior to what reported in previously published data [18] with regard to PFS at 3 years from transplant (42\%).

In contrast to the Italian study [22], which documented beneficial effects on survival only in patients achieving less than a VGPR after induction therapy, we did not observe statistically significant differences in OS and PFS on the basis of patients' response to induction treatment. However, when comparing OS and PFS curves according to patients' status prior to maintenance therapy (CR/ VGPR $v s$ PR), log-rank test was not significant ( $\mathrm{p}=0.067$ and 0.085 , respectively) probably due to the limited number of patients enrolled (Figure 9,10). Similarly, having found no significant differences in OS and PFS between patients who had undergone ASCT and patients that were not eligible for transplant ( $\mathrm{p}=0.0667$ and 0.085 , respectively, as shown in Figure 5,6), the efficacy of treatment regimens administered prior to maintenance therapy was most likely influenced by the small number of patients recruited, and especially by the smaller group that received ASCT (Table 2). The statistically significant advantage in TTNT seen in patients treated with ASCT (Figure 4) might be explained by the very good response of these patients to high-dose therapy, which resulted in a slower relapse/progression of disease and, therefore, a delayed need for retreatment.

We also analyzed intermittent low-dose thalidomide maintenance therapy on the basis of cytogenetics (assessed by both metaphase karyotyping and FISH): our findings are comparable with those reported in previously published data [4] in addition to a statistically significant advantage in patients with standard-risk cytogenetics vs patients classified as high-risk (Figure 7\&8).

Furthermore, the evaluation of toxicity confirmed that our intermittent low-dose thalidomide maintenance has a better tolerability profile: in our trial no patient had to stop thalidomide or have the dose reduced due to grade III-IV toxicity, in contrast to what reported in all above-mentioned studies where $30 \%$ to $80 \%$ of patients had to discontinue treatment because of adverse events, especially peripheral neuropathy. With our treatment protocol, minor polyneuropathy (grade I) occurred in just 1 patient, with no need to reduce and/or stop thalidomide. We observed 1 case of atrial fibrillation, 2 cases of grade II constipation, 8 cases of grade II fatigue, and no thromboembolic complications. The incidence and severity of toxicities seen in our trial were definitely inferior to those documented in previously published reports.

\section{Conclusion}

Our treatment protocol combining intermittent-low dose thalidomide (50mg/day for 2 weeks per month) with a fixed dose of 
dexamethasone (20mg/day for 4 days per month) as maintenance therapy had a positive impact on both PFS and OS, with results comparable or even superior to those reported in previously published researches. Our findings show that ISS stage, MM isotype and response to induction treatment do not significantly affect PFS and OS. Our therapeutic approach has also proved to be particularly beneficial to MM patients with favorable iFISH, as well as highly efficient in reducing treatment-related adverse events, especially peripheral neuropathy and venous thromboembolism, with an excellent safety profile: the quality of life of patients receiving this maintenance regimen was good and they were able to carry out normal daily activities, with no need for thalidomide dose reduction and/or therapy discontinuation.

Medical treatment protocols generally tend to employ novel agents, but it is also important to make optimal use of "older" agents that have been proven equally effective, if administered in the correct way.

Based on our findings and given its low toxicity and cost, maintenance therapy with intermittent low-dose thalidomide in combination with dexamethasone can be recommended in MM patients with no adverse iFISH profile. At present, it should therefore be taken into consideration when treating elderly patients with standard-risk cytogenetics.

\section{Authors' Contributions}

PG designed and supervised the study. CB, SF, and SM collected the data. VP and FT performed immunophenotypic analysis. AD and FM performed cytogenetic and FISH analyses. SA performed statistical analysis. AV and $\mathrm{MN}$ prepared thalidomide hard gelatin capsules. PG, PC and MR performed the research, analysed the data and wrote the paper. All authors reviewed and approved the final version of the manuscript.

\section{Acknowledgment}

The authors thank A.I.L. Ascoli Piceno "Alessandro Troiani" ONLUS for supporting this study and Federica Liberi for the English revision of the manuscript.

\section{Funding Information}

This study did not receive any specific grant from funding agencies in the public, commercial, or not-for-profit sectors.

\section{Compliance with Ethical Standards}

Research involving human participants: All procedures were conducted in accordance with the ethical standards of the institutional and/or national research committee and with the 1964 Helsinki declaration and its later amendments.

Informed consent: Informed consent was obtained from all individual participants included in the study.

\section{References}

1. Facon T. Maintenance therapy for multiple myeloma in the era of novel agents. Hematology Am Soc Hematol Educ Program. 2015; 2015: 279-285.

2. Brioli A, Tacchetti $P$, Zamagni E, Cavo M. Maintenance therapy in newly diagnosed multiple myeloma: current recommendations. Expert Rev Anticancer Ther. 2014; 14: 581-594.
3. McCarthy $\mathrm{PL}$, Palumbo A. Maintenance therapy for multiple myeloma Hematol Oncol Clin North Am. 2014; 28: 839-859.

4. Morgan GJ, Gregory WM, Davies FE, Bell SE, Szubert AJ, Brown JM, et al. National Cancer Research Institute Haematological Oncology Clinical Studies Group. The role of maintenance thalidomide therapy in multiple myeloma: MRC Myeloma IX results and meta-analysis. Blood. 2012; 119: 7-15.

5. Barlogie B, Desikan R, Eddlemon P, Spencer T, Zeldis J, Munshi N, et al. Extended survival in advanced and refractory multiple myeloma after singleagent thalidomide: identification of prognostic factors in a phase 2 study of 169 patients. Blood. 2001; 98: 492-494.

6. International Myeloma Working Group. Criteria for the classification of monoclonal gammopathies, multiple myeloma and related disorders: a report of the International Myeloma Working Group. Br J Haematol. 2003; 121: 749757.

7. Durie BG, Harousseau JL, Miguel JS, Bladé J, Barlogie B, Anderson K, et al. International Myeloma Working Group. International uniform response criteria for multiple myeloma. Leukemia. 2006; 20: 1467-1473.

8. Kaplan EL, Meier P. Nonparametric estimation from incomplete observations. J Am Stat Assoc. 1958; 53: 457-481.

9. Gray RJ. A class of K-sample tests for comparing the cumulative incidence of a competing risk. Ann Stat. 1988; 16: 1141-1154.

10. Berenson JR, Crowley JJ, Grogan TM, Zangmeister J, Briggs AD, Mills GM, et al. Maintenance therapy with alternate-day prednisone improves survival in multiple myeloma patients. Blood. 2002; 99: 3163-3168.

11. Shustik C, Belch A, Robinson S, Rubin SH, Dolan SP, Kovacs MJ, et al. A randomised comparison of melphalan with prednisone or dexamethasone as induction therapy and dexamethasone or observation as maintenance therapy in multiple myeloma: NCIC CTG MY.7. Br J Haematol. 2007; 136: 203-211.

12. Mandelli F, Avvisati G, Amadori S, Boccadoro M, Gernone A, Lauta VM, et al. Maintenance treatment with recombinant interferon alfa- $2 b$ in patients with multiple myeloma responding to conventional induction chemotherapy. N Engl J Med. 1990; 322: 1430-1434.

13. Cunningham D, Powles R, Malpas J, Raje N, Milan S, Viner C, et al. A randomized trial of maintenance interferon following high-dose chemotherapy in multiple myeloma: long-term follow-up results. $\mathrm{Br}$ J Haematol. 1998; 102: 495-502.

14. Barlogie B, Kyle RA, Anderson KC, Greipp PR, Lazarus HM, Hurd DD, et al. Standard chemotherapy compared with high-dose chemoradiotherapy for multiple myeloma: final results of Phase III US Intergroup Trial S9321. J Clin Oncol. 2006; 24: 929-936.

15. Attal M, Harousseau JL, Leyvraz S, Doyen C, Hulin C, Benboubker L, et al. Maintenance therapy with thalidomide improves survival in patients with multiple myeloma. Blood. 2006; 108: 3289-3294.

16. Stewart AK, Trudel S, Bahlis NJ, White D, Sabry W, Belch A, et al. A randomized phase 3 trial of thalidomide and prednisone as maintenance therapy after ASCT in patients with MM with a quality-of-life assessment: the National Cancer Institute of Canada Clinicals Trials Group Myeloma 10 Trial. Blood. 2013; 121: 1517-1523.

17. Lokhorst HM, van der Holt B, Zweegman S, Vellenga E, Croockewit S, van Oers $\mathrm{MH}$, et al. A randomized phase 3 study on the effect of thalidomide combined with adriamycin, dexamethasone, and high-dose melphalan, followed by thalidomide maintenance in patients with multiple myeloma. Blood. 2010; 115: 1113-1120.

18. Spencer A, Prince HM, Roberts AW, Prosser IW, Bradstock KF, Coyle L, et al Consolidation therapy with low-dose thalidomide and prednisolone prolongs the survival of multiple myeloma patients undergoing a single autologous stem-cell transplantation procedure. J Clin Oncol. 2009; 27: 1788-1793.

19. Palumbo A, Bringhen S, Liberati AM, Caravita T, Falcone A, Callea V, et al. Oral melphalan, prednisone, and thalidomide in elderly patients with multiple myeloma: updated results of a randomized controlled trial. Blood. 2008; 112: 3107-3114. 
20. Wijermans P, Schaafsma M, Termorshuizen F, Ammerlaan R, Wittebo $\mathrm{S}$, Sinnige $\mathrm{H}$, et al. Phase III study of the value of thalidomide added to melphalan plus prednisone in elderly patients with newly diagnosed multiple myeloma: the HOVON 49 Study. J Clin Oncol. 2010; 28: 3160-3166.

21. Ludwig H, Adam Z, Tóthová E, Hajek R, Labar B, Egyed M, et al. Thalidomide maintenance treatment increases progression-free but not overall survival in elderly patients with myeloma. Haematologica. 2010; 95: 1548-1554.
22. Offidani M, Corvatta L, Polloni C, Piersantelli MN, Gentili S, Galieni P, et al. Thalidomide-dexamethasone versus interferon-alpha-dexamethasone as maintenance treatment after ThaDD induction for multiple myeloma: a prospective, multicentre, randomised study. $\mathrm{Br} \mathrm{J}$ Haematol. 2009; 144: 653659
Ann Hematol Oncol - Volume 6 Issue 2 - 2019

ISSN : 2375-7965 | www.austinpublishing group.com

Galieni et al. () All rights are reserved
Citation: Galieni P, Caraffa P, Bigazzi C, Falcioni S, Mazzotta S, Pezzoni V, et al. Intermittent Low-Dose Thalidomide Plus Dexamethasone as Maintenance Therapy in Patients with Multiple Myeloma. Ann Hematol Oncol. 2019; 6(2): 1235 